\title{
Dimensionality, topology, energy, the cosmological constant, and signature change *
}

\author{
Franz Embacher \\ Institut für Theoretische Physik \\ Universität Wien \\ Boltzmanngasse 5 \\ A-1090 Wien \\ E-mail: fe@pap.univie.ac.at \\ UWThPh-1995-11 \\ gr-qc/9504040
}

\begin{abstract}
Using the concept of real tunneling configurations (classical signature change) and nucleation energy, we explore the consequences of an alternative minimization procedure for the Euclidean action in multiple-dimensional quantum cosmology. In both standard Hartle-Hawking type as well as Coleman type wormhole-based approaches, it is suggested that the action should be minimized among configurations of equal energy. In a simplified model, allowing for arbitrary products of spheres as Euclidean solutions, the favoured spacetime dimension is 4 , the global topology of spacelike slices being $\mathbf{S}^{1} \times \mathbf{S}^{2}$ (hence predicting a universe of Kantowski-Sachs type). There is, however, some freedom for a Kaluza-Klein scenario, in which case the observed spacelike slices are $\mathbf{S}^{3}$. In this case, the internal space is a product of two-spheres, and the total space-time dimension is $6,8,10$ or 12 .
\end{abstract}

\footnotetext{
*Work supported by the Austrian Academy of Sciences in the framework of the "Austrian Programme for Advanced Research and Technology".
} 


\section{Introduction}

This paper is devoted to a suggestion concerning the procedure of minimizing the Euclidean action, in particular for models in which the cosmological constant $\Lambda$ is effectively not a fundamental quantity but can be treated as a degree of freedom by its own. Such models are encountered within the standard no-boundary approach of Hartle and Hawking [1] [2] as well as in the formalism including wormeholes and baby universes, due to Giddings and Strominger [3], Coleman [4] [5] and others. Usually, in order to find the dominant contributions to the underlying path integral, the Euclidean action (evaluated at solutions of the field equations) is minimized at constant values of $\Lambda$ (which yields $\mathbf{S}^{4}$ in the four-dimensional pure gravity case). In any framework based on the no-boundary proposal, the minimizing configuration describes the most probable initial condition for the classical universe at nucleation, and is associated with the "probability" $P \sim \exp (-I)$. In the approaches including wormholes, the configurations miminizing the effective Euclidean action represent the bunch of universes connected with each other. Their action determines the measure for the wormhole parameters, the most famous application thereof being Coleman's argument that $\Lambda=0$ in all observations. We will always have these two types of frameworks in mind, although the relevant objects and procedures may play a different role therein. The overall philosophy of these approaches is the same [5]. However, an essential technical difference between them is that the models including wormholes and baby universes involve approximations that make them less applicable to quantum cosmological situations (i.e. to questions concerning the "history" of the very early universe, as e.g. whether it can nucleate with $\Lambda \neq$ 0 ). In contrast, the (non-wormhole) Hartle-Hawking type models are formulated in terms of cosmologically relevant quantities from the outset, and "nucleation of the universe" is quite clearly associated with a wave function that behaves exponential in some domain of superspace and oscillatory everywhere else. Nevertheless, we will formulate our proposal with the motivation that it might be applicable for both.

It has already been observed that admitting configurations of arbitrary dimensions leads to the prediction that the observed dimension is very large. Several modifications have been suggested in order obtain a framework that predicts the observed space-time to be four-dimensional (see e.g. Refs. [6]-[8]). Here, we would like to suggest an alternative point of view, based on a modified concept of "Euclidean solutions". Usually, the action is minimized among solutions of the Euclidean field equations, each solution (instanton) being defined on a compact manifold $\mathcal{M}$ 
without boundary. Our starting point is that an instanton (sloppily denoted by $\mathcal{M}$ ) is not directly related to physical observables. On the other hand, the nucleation of the universe as a tunneling phenomenon can be described in terms of a "real tunneling geometry", i.e. a classical field configuration whose metric signature changes from Euclidean to Lorentzian type across a hypersurface $\Sigma$, which plays the role of the initial spacelike slice of a classical universe [9]. The Euclidean part of the corresponding manifold represents the "tunneling from nothing", whereas "at" $\Sigma$ the classical (Lorentzian) time emerges. This situation can be encoded in terms of a purely Euclidean configuration, defined on $\mathcal{M}$, together with a hypersurface of zero extrinsic curvature (or more general: a "symmetric" hypersurface). These issues are reviewed in Section 2. Our proposal amounts to replace $\mathcal{M}$ by the pair $(\mathcal{M}, \Sigma)$ as the class of objects to be compared by means of their Euclidean action. (In a wormhole-based framework, the interpretation of $\Sigma$ is less straightforward than in (non-wormhole) Hartle-Hawking type quantum cosmology, but at least formally it provides an $(n-1)$-dimensional "space" $\Sigma$ associated with an $n$-dimensional instanton $\mathcal{M})$. Interpreting $\Lambda$ as a vacuum energy density, we associate with any such pair $(\mathcal{M}, \Sigma)$ a nucleation energy $E \sim \Lambda \times$ volume of $\Sigma$ (including additional matter terms in general). It is then suggested that - as an alternative to the standard procedure - the minimization of the Euclidean action could be performed at constant $E$. This scheme enables one to extract probabilities for (or at least a relative order among) the real tunneling configurations in different dimensions. As a consequence, low dimensions (large energies) are favoured, and the most probable topology of spacelike slices may be predicted even in multiple-dimensional theories. In Section 3 , we formulate our proposal and give some heuristic motivations. We should emphasize here that all approaches based on the value of the action on solutions of the Euclidean field equations (i.e. the "on shell"-action) provide only approximations to the full quantum theory for certain types of questions, the procedure we suggest being included of course. What we have in mind in the present paper are questions concerning the "large-scale" variables, in particular the dimension and topology of space and space-time. Despite the inclusion of "effective" phenomena (such as the cosmological constant), the underlying small-scale dynamics has largely been truncated, so that e.g. the issue of decoherence [10]-[1] is inaccessible within such a framework. Neverthless, it is just this issue that accounts for the selection of a real, classical universe. We understand our suggestion as a possible effective imprint of the "true" underlying quantum theory on the approximate level of a minimize-theaction procedure. 
In order to have a computable scenario at hand, we present a somewhat simplified model in Section 4, neglecting all matter contributions except for the cosmological constant, and admitting arbitrary products of spheres in any dimension as the fundamental configurations $\mathcal{M}$. The associated hypersurfaces $\Sigma$ are provided by the equators of the factor spheres. The first step in minimizing the action, keeping the energy $E$ fixed as well as the dimension $n$, is carried out in Section 5 . We encounter a sequence of minimizing configurations, denoted by $\mathcal{K}_{n}$, each being a product of two-spheres with some $p$-sphere $(p \sim \sqrt{n}$ for large $n)$. It turns out that for large $E$ the favoured dimension is $n=4$, the corresponding spacelike slice $\Sigma$ being $\mathbf{S}^{1} \times \mathbf{S}^{2}$, i.e. of Kantowski-Sachs type. If the minimization is only approximate (caused by the details of some decoherence mechanism or by an effect at the wormhole scale), there is some freedom for a Kaluza-Klein scenario with spacelike slice $\Sigma=\mathbf{S}^{3}$ and total dimension $n=6,8,10$ or 12 . The internal (compactified) space is a product of two-spheres. These results provide the main arguments in favour of our proposal. In addition, we discuss the configurations at low energies, and find evidence for a higher-dimensional phase of the universe "before" nucleation.

In Section 6, we demonstrate the (appearently quite generic) feature that minimization at fixed but large $E$ leads to low dimensions, whereas in Section 7 we show that among the configurations selected already, those with large $E$ are favoured. This indicates that nucleation can be expected at large energies, and provides the completion of the arguments leading to the prediction of the above-mentioned configurations. The role of $E$ being large at nucleation is formally related to the smallness of $\Lambda$ in the standard wormhole theory. Thus we mention that our framework might be of some significance for the late (classical) universe as well, $(E, \Sigma)$ representing the actual energy and the spacelike slices. If this is true, our proposal might anticipate some generalization of Coleman's wormhole model for the case of a cosmological scenario (and probably the inclusion of some large-scale decoherence mechanism). We point out that in a somewhat effective sense our action for the late universe coincides with Coleman's (equation (7.3) below). In Section 8, we speculate about a modification of the fundamental gravity action by boundary terms that could render the real tunneling configurations the basic objects without imposing any ad hoc hypothesis. Finally, we mention the generalization of an alternative minimization procedure within our framework, based on a related idea originally due to Gasperini [7], using a different concept of "energy". It predicts space-time to be $\mathbf{R} \times \mathbf{S}^{3}$, although its applicability might be limited to (products of) spheres.

To summarize, the most direct way of extracting a favoured classical space- 
time from our model gives $\mathbf{R} \times \mathbf{S}^{1} \times \mathbf{S}^{2}$ in $n=4$, but it is also consistent with $\mathbf{R} \times \mathbf{S}^{3} \times$ internal space in $n=6,8,10$ or 12 . The computations leading to these results are mainly contained it Section 5. (We should add that it is presumably quite easy to write down a model containing a slightly more sophisticated matter sector, such that the favoured classical space-time is $\mathbf{R} \times \mathbf{S}^{3}$ - which is certainly the most appealing configuration). Thus, from the point of view of dimensionality, our proposal reproduces a reasonable behaviour of the early universe. We must leave it open whether, apart from this issue, it might as well be helpful with regard to the conceptual problems of quantum cosmology.

\section{Euclidean action and real tunneling configura- tions}

One of the drawbacks in Euclidean quantum gravity is the fact that the Euclidean action is not bounded from below. Thus, a path integral over "all" compact Euclidean (i.e. positive signature) metrics diverges badly. There have been techniques developed to impose a separate analytic continuation in the conformal freedom 12 - 15. Within such a scheme, the dominant contributions in the path integral are provided by the stationary points of the action (i.e. solutions to the Euclidean field equa-

tions) that are not necessarily local minima. The according procedure consists of evaluating the Euclidean action "on shell". Thus restricted, the gravitational action (including a fixed positive cosmological constant) is bounded from below [16], and the particular solution that minimizes the action is expected to be the dominating one.

The Euclidean action in an abitray number of dimensions reads

$$
I=-\frac{1}{16 \pi G_{n}} \int_{\mathcal{M}} d^{n} x \sqrt{g}(R-2 \Lambda)-\frac{1}{8 \pi G_{n}} \int_{\partial \mathcal{M}} d^{n-1} x \sqrt{h} K+I^{\text {else }},
$$

where $G_{n}$ is the $n$-dimensional gravitational constant, and $I^{\text {else }}$ stands for contributions from matter fields (and possibly higher order curvature terms). An action of this type occurs in different frameworks, either representing a fundamental theory including various matter interactions, or just as an effective action obtained after (some or all) non-gravitational degrees of freedom have been integrated out. If the possibility of wormholes and topology fluctuations at small scales is included, the coupling constants (in particular the cosmological constant $\Lambda$ ) depend on the 
wormhole parameters and may in some sense be treated as degrees of freedom by their own. The stationary points of $I$ are the solutions of the Euclidean Einstein equations

$$
R_{\mu \nu}-\frac{1}{2} g_{\mu \nu} R=-\Lambda g_{\mu \nu}+8 \pi G_{n} T_{\mu \nu}^{\mathrm{else}} \equiv 8 \pi G_{n} T_{\mu \nu}^{\mathrm{tot}}
$$

If $I$ explicitly contains matter fields $\Phi$, their corresponding equations of motion (obtained from $\delta I=0$ along with $(2.2)$ ), are taken into account as well. Assuming $\Lambda>0$ and a reasonable behaviour of the matter sector, there are two different types of solutions $(\mathcal{M}, g, \Phi)$ of interest. Either $\mathcal{M}$ is a compact manifold without boundary, (in which case we call the configuration an "instanton") or $\mathcal{M}$ is compact and has a boundary $\mathcal{N}$ at which $(h, \phi)$ - the appropriate restrictions of $(g, \Phi)$ - are prescribed. The instanton configurations of the effective action arise quite directly in the evaluation of the path integral when wormholes and baby universes are included [3] [4] [5]), whereas the second type of solutions is due to the conventional situation in quantum cosmology, when the wave function of the universe $\psi[h, \phi]$ is computed in the semiclassical approximation [1] [2]. For pure gravity $\left(T_{\mu \nu}^{\text {else }}=0\right)$, the most symmetric instanton is the round sphere $\mathbf{S}^{n}$ with radius $a$ given by $a^{2}=(n-1)(n-$ $2) / 2 \Lambda$. The action thereof provides a general lower bound, i.e. it is less than $I(\mathcal{M}, g)$ evaluated at any other pure gravity instanton in the same dimension and with the same $\Lambda$ [16].

With any instanton configuration $(\mathcal{M}, g, \Phi)$, one usually associates a weight $\exp (-I)$. This is often treated as if it was an amplitude or probability (see e.g. Refs. [6], [17] and [18]). In the path integral approach to quantum cosmology based on the no-boundary proposal by Hartle and Hawking [1] [2] (which is just the formalization of the requirement that only compact metrics are involved) the dominant contribution to $\psi[h, \phi]$ is not provided by instantons but by solutions with boundary. However, the instantons describe the nucleation ("tunneling from nothing") of the universe. In the simplest $(n=4 \mathrm{FRW})$ minisuperspace example with $T_{\mu \nu}^{\text {else }}=0$ and $\Lambda$ fixed, the only instanton is the four-sphere with radius $a\left(a^{2}=3 / \Lambda\right)$. Any three-sphere with radius $b<a$, when placed into the instanton manifold as a hypersurface, divides $\mathbf{S}^{4}$ into two pieces, the action $I_{H H}(b)$ of the smaller one giving rise to the (approximate) wave function $\psi(b) \sim \exp \left(-I_{H H}(b)\right)$. For $b>a$, the condition that $\mathbf{S}^{4}$ contains a larger $\mathbf{S}^{3}$ can only be met by analytic continuation to complex numbers, and $\psi(b)$ becomes oscillating, i.e. provides the semiclassical wave function for the evolution of the (Lorentzian) universe [回]. Thus, the universe nucleates (becomes "real" or classical) at a scale factor $b \approx a$. Since $\psi(a) \sim \exp (-I / 2)$, where 
$I$ is the total Euclidean action of the instanton $\mathbf{S}^{4}$, the quantity $P \sim \exp (-I)$ may be referred to as the (approximate) "nucleation probability". In similar situations, but with $\Lambda$ being only an effective vacuum energy density (e.g. induced by a scalar field, $\Lambda=8 \pi G_{n} V(\phi)$, in the approximation that the dynamics of $\phi$ is ignored), the nucleation probabilities according to different values of $\Lambda$ (or to even to different wave functions) are directly compared, and supposed to yield an estimate for the most probable classical initial configuration (see e.g. Refs. [19], [9] and [20]). A more general concept of the class of all instantons which provide a "nucleation seed" for the universe within the no-boundary proposal is provided by the "real tunneling configurations" (see below).

In a model including wormholes ( $I$ denoting the effective action), the weight $\exp (-I)$ becomes once more exponentiated, giving rise to a contribution $\exp (-I) \exp (\exp (-I))$ to the measure for the wormhole parameters [5]. The cosmological constant thereby effectively represents the small scale topology fluctuations (cf. Ref. [21]). The minimizing instanton configuration provides the dominant (in some situations even the only) contribution to the measure for the wormhole parameters. This is the basis for Coleman's argument that the connection of our universe with other universes by wormholes forces the obverved $\Lambda$ to be exactly zero (as soon as the universe is larger than the wormhole scale). The technical reason is that for fixed $\Lambda$ the action is minimized by the four-sphere and takes the value $I=-3 \pi / G \Lambda$, which in turn becomes $-\infty$ as $\Lambda \downarrow 0$. Originally, Coleman supposed the integration over all matter fields to have been carried out already [5], although later authors kept some or all non-gravitational degrees of freedom in the effective action (see e.g. Refs. [6] and [8]), in particular if non-trivial matter vacuum configurations exist. In any case, the action is of the type (2.1), possibly with $I^{\text {else }}$ being disregarded or containing only higher order curvature terms. Minimization of the action at constant $\Lambda$ again gives (for $n=4$ and pure gravity degrees of freedom contained in $I$ ) the instanton $\mathbf{S}^{4}$, and only thereafter the dependence of $\Lambda$ on the wormhole parameters is taken into account (leading to the $\delta(\Lambda)$-peak in turn). However, alternative minimalization procedures have been suggested. In particular, if arbitrary dimensions are admitted by the underlying theory, the minimization of $I$ at constant $\Lambda$ leads to $n \rightarrow \infty$. Gasperini [7] considered $n$-spheres as instantons in arbitrary dimensions and proposed that the cosmological constant depends on the dimension $n$. He minimized the action at constant values of the (formal) energy $U$ contained in the Euclidean geometry on the $n$-sphere (it is defined by $\Lambda / 8 \pi G_{n}$ times the volume of a ball in $\mathbf{R}^{n-1}$ with the same radius as the instanton $\mathbf{S}^{n}$, and is proportional to $\partial I / \partial \beta$ as 
formally computed in the thermodynamic approach to Euclidean quantum gravity; in Section 8 we comment on this approach in more detail). His conclusion that flat space-time is four-dimensional is appealing, but the relation $U$ to an observed energy is not clear. Other approaches to the dimensionality problem based on the Euclidean action may be found in Refs. [22], [6] and [8].

In what follows we envisage models whose $\Lambda$ is enough of an effective phenomenon such that it can be treated as an independent variable when the action is minimized. In the wormhole formalism, this is obviously the case, but one may encounter similar situations within the Hartle-Hawking framework as well (e.g. if $\Lambda=8 \pi G_{n} V(\phi)$ or if some underlying dependence of $\Lambda$ on additional variables - with or without dynamics - is assumed; cf. Refs. [17] and [18]). One may also consider a third kind of models - ranging somewhere in between the Hartle-Hawking and the Coleman type appoaches - by naively assuming that $\Lambda$ effectively encodes the small-scale "foam"-like structure of space-time, but without admitting wormholes that connect different universes [21]. This just renders $\Lambda$ an own degree of freedom as well. With regard to to conceptual questions, this type of models is closer related to the HartleHawking formalism, into which it shall henceforth be included. Traditionally, as mentioned, in all these cases the minimization of $I$ (e.g. with respect to topology) is first carried out at constant $\Lambda$ (see e.g. Refs. [5] and [9]). If we parametrize the general instanton solution to a given $\Lambda$ symbolically by $\gamma$ (which denotes a collection of a huge number of parameters to characterize the solutions, including their dimension and topology), the action of arbitrary instantons may be written as $I_{\Lambda}(\gamma)$. Varying with respect to $\gamma$ at constant $\Lambda$ yields a minimizing configuration, denoted by $\gamma(\Lambda)$. (If $n=4$ is kept fixed from the outset, $\gamma(\Lambda)$ just denotes the instanton $\mathbf{S}^{4}$ with $a^{2}=3 / \Lambda$ ). In a second step, one associates with $\Lambda$ the number $I_{\Lambda}(\gamma(\Lambda))$ which can be minimized again (to give $\Lambda \downarrow 0$ in general).

The aim of this paper is to suggest a modified way of performing the minimization of $I$. The role of instantons as "nucleation seeds" in the no-boundary framework has already been mentioned for the case of the FRW minisuperspace model. The initial classical spacelike slice is provided by the three-sphere with radius $a$, hence the equator of the instanton $\mathcal{M}=\mathbf{S}^{4}$. Let us denote this hypersurface by $\Sigma$. In general, there is a variety of pairs $(\mathcal{M}, \Sigma)$, each being a candidate for inducing nucleation ("at" $\Sigma$ as the initial spacelike slice) and each being associated with the (approximate) nucleation probability $P \sim \exp (-I)$.

The idea of associating with a Euclidean configuration $\mathcal{M}$ a hypersurface $\Sigma$ can be made more precise by introducing the concept of "real tunneling geometries" 
[9] (or "real tunneling configurations", to be more general) and "classical signature change" ( see Refs. [23]-30]). In analogy with the usual way one treats tunneling phenomena in quantum mechanics, the process of "tunneling from nothing" of the universe can be described by a Euclidean solution of the field equations that matches smoothly the subsequent classical evolution in Lorentzian time. The corresponding construction is a configuration $(g, \Phi)$ on some manifold $\mathcal{M}_{\text {real tunneling }}$ such that across a hypersurface $\Sigma$ the signature of the metric changes from Euclidean $(+,+\ldots,+)$ to Lorentzian $(-,+\ldots,+)$ type.$\Sigma$ is spacelike with respect to the Lorentzian side. The Euclidean part $\mathcal{M}_{\text {Eucl }}$ is compact (its boundary of course being $\Sigma$ ), and the field equations (including Einstein's equations, which are defined independent of the metric signature) are satisfied in the two single-signature

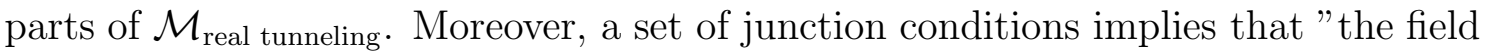
equations" are satified "at" the changing hypersurface $\Sigma$ as well: These conditions require the configuration as a whole to be a stationary point of the action (which is in this context considered as a genuine complex quantity, evaluated at genuine complex field configurations [9]). They essentially state that the extrinsic curvature induced by the metric $g$ on $\Sigma$, along with the normal (affine parameter) derivatives of certain matter field components must vanish when computed with respect to either (Lorentzian or Euclidean) side (see e.g. Refs. [9] and [23]). In other words, $\Sigma$ must carry "time-symmetric" initial-data. In terms of a slicing such that $\Sigma$ is

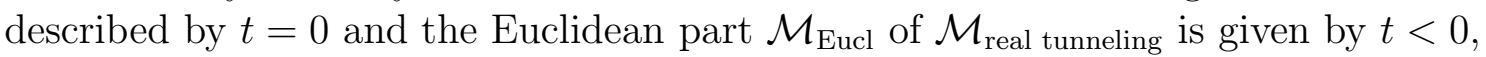
the junction conditions imply the following: If the Euclidean "time" evolution starts from some value $t<0$ and is re-performed, but without changing the signature at $t=0$, then the Euclidean solution emerging for $t>0$ is just the time-reversed (reflected) version of the solution in the region $t<0$, the total result being a purely Euclidean solution on a compact manifold $\mathcal{M}$ without boundary (hence an instanton), carrying a "time"-symmetric hypersurface $\Sigma$ (In the notation of Ref. [9], $\mathcal{M}$ is written as $\left.2 \mathcal{M}_{\text {Eucl }}\right)$. The quotation marks indicate that $t$ is the Euclidean time on $\mathcal{M}$. We will simply call $\Sigma$ a symmetric hypersurface. Likewise, there is a purely Lorentzian solution with a hypersurface $\Sigma$ carrying time-symmetric data. The man-

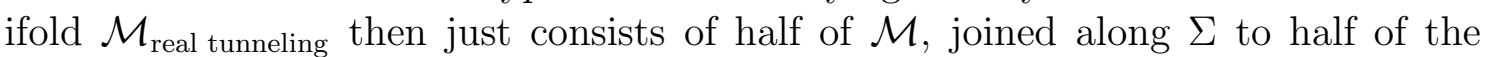
corresponding Lorentzian manifold. If the above-mentioned "time" coordinate $t$ is chosen appropriately, the two parts of $\mathcal{M}_{\text {real tunneling }}$ are related by a Wick rotation in $t$ [9]. This representation of the "brith of the universe" may be referred to as strong signature change and should not be confused with weak signature change, which is an attempt to define an alternative theory of gravity [30]. 
To summarize, a real tunneling configuration is provided by an instanton $(\mathcal{M}, g, \Phi)$, together with a symmetric hypersurface $\Sigma$. Any such configuration describes a possible nucleation of the universe, where $\Sigma$ and its data $(h, \phi)$ provide the initial conditions for the subsequent (classical) time evolution [9]. The Euclidean action evaluated over the tunneling part is $I / 2$, where $I$ is the total action of the instanton (the boundary term in (2.1) being obsolet just because the extrinsic curvature on $\Sigma$ vanishes). The according nucleation probability is $P \sim \exp (-I)$.

The simplest example of a signature changing solution of the pure Einstein equations with a positive cosmological constant $\Lambda$ is provided by cutting an $n$-sphere along its equator and joining it to the corresponding half of a de Sitter space $\mathbf{H}^{n}$ (cf. Refs. [31] and [9]). The "radius" is related to the cosmological constant by $a^{2}=(n-1)(n-2) / 2 \Lambda$, and the real tunneling geometry is given by the metric

$$
d s^{2}=-\operatorname{sgn}(t) d t^{2}+b(t)^{2} d \sigma_{n-1}^{2}
$$

where $t$ is ranging from $-a \pi / 2$ to $\infty$, and $d \sigma_{p}^{2}$ is the round metric on the unit $p$-sphere. Furthermore,

$$
b(t)=\left\{\begin{array}{cc}
a \cos (t / a) & -a \pi / 2<t<0 \\
a \cosh (t / a) & 0<t .
\end{array}\right.
$$

Let us denote the space thus defined by $\mathbf{K}^{n}$. The hypersurface $\Sigma=\mathbf{S}^{n-1}$ is given by $t=0$. The region $t<0$ is half of $\mathbf{S}^{n}$, the region $t>0$ is half of $\mathbf{H}^{n}$. This configuration represents the quantum tunneling process occuring in the $n=4$ FRW minisuperspace model. The genuine quantum state of the universe (i.e. the state in which it is in the classically forbidden region "smaller than the minimum de Sitter radius $a "$ ) is visualized as the Euclidean part, whereas the Lorentzian part represents the subsequent classical evolution.

The physical significance of $\Sigma$ as the initial classical hypersurface is rather clear if the underlying concept is the no-boundary wave function of the universe. However, we would like consider the identical construction in the case of the effective action appearing in wormhole-based models as well. There, the relation between Euclidean (instanton) configuration and observable quantities is less direct. In some formal sense, the instantons are associated with a variety of universes, connected with each other by wormholes. However, the precise role played by these configurations depends on the issue of how to regularize and compute the path integral (cf. e.g. Ref. [32]), and thouch upon deeper conceptual questions of quantum gravity. 
It is in particular the appearance of arbitrarily large universes that causes the need for a cutoff [5], and that might look unappealing within the context of cosmology. It is conceivable that in some quantum cosmological modification of Coleman's theory (taking into account the "smallness" of the universe, or universes) the instantons of the effective action play a role comparable to the Euclidean configurations dominating the wave function in the no-boundary approach and giving rise to the concept of nucleation. Thus, we consider the pairs $(\mathcal{M}, \Sigma)$ - rather than $\mathcal{M}$ alone - as the relevant objects in this case as well. We admit that it is not obvious whether such a modification would apply only to the cosmological context, and whether it might be relevant for today's (large) universe as well (e.g. in the sense that a scale factor $a$ of $\Sigma$ just represents a quantum contribution to the actual scale factor observed; we will speculate on this at the end of Section 7).

Having adopted the concept of real tunneling configurations $(\mathcal{M}, \Sigma, g, \Phi)$ as relevant quantities in models described by an action of the type (2.1), we should state that an instanton may admit several non-equivalent symmetric hypersurfaces $\Sigma$. On the other hand, not any $(\Sigma, h, \phi)$ will be associated with an instanton. Hence, in our symbolic notation, the set of all $\mathcal{M}$ is not identical to the set of all $(\mathcal{M}, \Sigma)$. However, we expect all dominant configurations to be contained in both classes, and different situations to be suppressed by the path intgral.

\section{Nucleation energy}

We are now in a position to develop our proposal. If $\Lambda$ is thought of as "the vacuum energy density", one might like to know whether this quantity refers to some "space", in which a corresponding amount of energy is distributed. We follow our line of reasoning and treat the real tunneling configurations as the relevant ones. In view of the nucleation scenarios, it is natural to associate the "energy density" referred to as $\Lambda$ (plus possible contributions from the matter fields $\Phi$ ) with the initial classical hypersurface $\Sigma$. Let us denote by $E$ the total energy contained in the matter fields (including $\Lambda$ ) thus defined. Its density is given by

$$
\rho=\frac{\Lambda}{8 \pi G_{n}}-T_{\mu \nu}^{\mathrm{else}} n^{\mu} n^{\nu} \equiv-T_{\mu \nu}^{\mathrm{tot}} n^{\mu} n^{\nu}
$$

where $n^{\mu}$ is the unit normal to $\Sigma$. (In the formula above, the energy momenum tensor is the Euclidean one, and $n^{\mu} n_{\mu}=1$, hence the unusual sign. However, 
this expression has the advantage of being defined with respect to the Euclidean configuration only). The total energy associated with this density is thus

$$
E=\int_{\Sigma} d^{n-1} x \sqrt{h} \rho \equiv \frac{1}{8 \pi G_{n}} \mathcal{V}_{\Sigma} \Lambda-\int_{\Sigma} d^{n-1} x \sqrt{h} T_{\mu \nu}^{\mathrm{else}} n^{\mu} n^{\nu}
$$

where $\mathcal{V}_{\Sigma}$ is the volume of $\Sigma$ as an $(n-1)$-manifold.

If $\Lambda$ is allowed to take arbitrary values, (which is our assumption), this last equation can be solved for $\Lambda$ in terms of $E$ (and the additional variables, encoding all further properties, including the dimension and topology). Let us symbolically denote by $\alpha$ the variables characterizing the field configuration on $(\mathcal{M}, \Sigma)$ at fixed $E$. Thus, with each real tunneling configuration we associate the corresponding value for $E$, and one arrives at an alternative expression for the action, denoted by $I_{E}(\alpha)$. This might be viewed as a trivial change of variables, but we should keep in mind that the equation relating $\Lambda$ and $E$ involves all the variables $\alpha$. (The variables $\alpha$ and $\gamma$ partially coincide, but are not identical).

Let us proceed heuristically. Nucleation (i.e. the emergence of a superposition of semiclassical universes) is probably related to decoherence [10]-11] (i.e. the actual selection of one universe that is classical at least with respect to the large-scale variables such as dimension and topology). We do not know the details of this selection process. (Clearly, it cannot be understood in terms of a formalism based mainly on the value of the action for instantons and real tunneling configurations. As a consequence, decoherence shows up effectively as a process that might be dealt with heuristically and formally, but whose origin is external to our framework). It should not be a surprise if the emergence of classical time is related fundamentally to its conjugate quantity, energy. Minimization of the action is certainly one basic principle to characterize this mechanism. However, the action usually does not admit a well-defined minimizing configuration as long as all its variables are allowed to vary arbitrarily. One possibility to guess a futher principle accoding to which the selection operates is to conjecture that the "competition" among various configurations favours those at equal energy prior to those at different energy.

One may look at this in an even more heuristic way: There is some amount of energy $E$ associated with the initial field configuration on $\Sigma$. Gasperini [7] mentioned the idea that there could have been only a certain amount of energy "available" for the formation of geometrical structures. However, in a sense, the total energy of a closed universe is zero. Hence, $E$ is precisely compensated by an amount of energy 
$-E$ contained in the gravitational field. Thus one can look at the mechanism that drives the universe classical as a process with energy balance $0 \rightarrow E-E$. If this transition of "zero energy" into two precisely cancelling contributions is somehow constrained in a quantum state, the sub-spaces at constant $E$ in the space of all configurations attain a preferred role. Likewise, one might think about $E$ to be "conserved" during transitions between configurations with different dimension and topology.

Thus, in technical terms, we suggest to minimize the action $I_{E}(\alpha)$ at fixed $E$ (instead of fixed $\Lambda$ ), thereby obtaining a set of minimizing configurations, symbolically denoted by $\alpha(E)$. The subsequent minimization of $I_{E}(\alpha(E)$ ) (in general leading to $E \rightarrow \infty$, as a sort of analogy to $\Lambda \downarrow 0$ ) indicates that the unicerse becomes classical at large $E$. We do not know exactly at which value of $E$ this happens, but in the simplified model to be considered in the remaining Sections, we will encounter a good candidate. Moreover, the configurations for large $E$ will turn out to be fourdimensional. In contrast, the configurations $\alpha(E)$ for small $E$ have large $n$ and represent the genuine multiple-dimensional quantum state of the early universe.

As a consequence of the procedure we suggest, $\Lambda$ effectively becomes dependent not only on the dimension (as was anticipated before [7]) but on the particular configuration itself. In some sense, our idea is that despite the key role played by the cosmological constant at a more fundamental level, the mechanism that causes the universe to become classical (at large scales) might be related closer to the nucleation energy than to $\Lambda$. The formal advantage of any approach based on real tunneling configurations is that the concept of energy $(E)$ and space $(\Sigma)$ is associated with a certain Euclidean solutions of the field equations.

So far we have laid emphasis on nucleation scenarios. A similar procedure may also be tried within a wormhole-based model, although in a more formal way since the significance of $\Sigma$ and $E$ for the Euclidean configurations representing the bunch of universes is not as transparent as in the Hartle-Hawking framewok. However, as already mentioned, it might be that some quantum cosmological modification of Coleman's appoach looks formally quite similar. The modification of the standard procedures implied by our suggestion, if it is taken over to the wormhole model, may be illustrated by Coleman's proposal that the value of certain coupling constants ("constants of nature", they are contained in our $\gamma$ ) may be determined by minimizing the effective action $I_{\Lambda}(\gamma)$ at small constant $\Lambda \downarrow 0$ [5]. In our approach, this is replaced by minimizing $I_{E}(\alpha)$ (the coupling constants now being contained in $\alpha$ ) at large constant $E \rightarrow \infty$. As far as the dimension is concerned, our approach 
(like Gasperini's [7]) will predict $n=4$ as the lowest possible value, whereas minimization at constant $\Lambda$ would lead to $n \rightarrow \infty$. It is not clear conceptually, however, whether the limit $E \rightarrow \infty$ is to be understood as the prediction that space is (tends to be) flat, and whether at least the topoloy and dimension of $\Sigma$ have some physical significance apart from the issue of nucleation. (Also, it is not clear to what extent $E$ and $\Sigma$ refer to "our" universe, or to the bunch of universes that exist in the wormhole-based models). The suggestion we made might be reasonable only for the early universe which is about to nucleate. In any case, as soon as the size of the universe exceeds the wormhole scale, Coleman's original theory should begin to apply, possibly somehow "decoupled" from the large-scale phenomena such as the dimension and topology of space. Also, whether our procedure can effectively be made equivalent to the standard wormhole model as far as the late universe is concerned (e.g. whether the quantities $E$ and $\Sigma$ can be given a conceptually convincing role in a path integral), must be left open. We will find a certain hint for further speculations in Section 7 (equation (7.3)).

Due to our lack of knowlegde about the details of the underlying nucleation or large-scale decoherence mechanism, the probabilistic interpretation might apply only approximately. In other words, the process of "freezing out" a particular $(\Sigma, h, \phi)$ (to play the role of the classical initial data) can reasonably be expected to select some configuration whose action is small but maybe non-minimal. In a model containing wormholes, such a mismatch between propabilistic law and actual dynamics may also be due to an effect induced by the existence of a wormhole scale (whose magnitude is unknown, cf. Ref. [5]). One may imagine for example some mechanism that "freezes out" a classical scale factor just above the wormhole scale, and hence at an energy scale $E$ that we do not know exactly. The suppression of a subsequent tunneling (dimensional transition) into a lower dimensional state would of course have to be explained in such a scenario. We shall take into account this possibility when interpreting our numerical results in Section 5.

In the whole of this paper we ignore higher loop contributions. It is likely that more appropriate (and accurate) results can be achieved by redefining the nucleation probabilities as $P \sim A \exp (-I)$, where $A$ is the one-loop prefactor arising from fluctuations around the Euclidean configuration (see e.g. Refs. [12] and [33]). Hence, our results certainly become unreliable as $E$ gets very small. However, including these corrections, we do not expect the principle structure of the analysis and its conceptuals difficulties to change dramatically. Also, we do not consider the approach to quantum cosmology due to Vilenkin and Linde, based on the so-called 
"tunneling wave function" of the universe [31], [34]-[36]). In a sense, the Euclidean action enters this formalism with different sign, so that one ends up with probabilities $P \sim \exp (I)$. Naively replacing the minimization by maximization of $I$ in our framework, one would obtain arbitrarily high nucleation dimensions to be favoured. Let us just mention that this could be avoided within our proposal by an anomalous behaviour of the gravitational constant for $n \rightarrow \infty$ (cf. equation (3.3) below and the discussion of the $\kappa_{n}$ at the beginning of Section 6).

Moreover, we will neither specify the particular matter content of the fundamental theory, nor of the effective action in the wormhole-based models. However, as far as nucleation is concerned, the initial configuration $(\Sigma, h, \phi)$ carries some (effective) non-zero cosmological constant $\Lambda$. In the Hartle-Hawking formalism without wormholes, $\Lambda$ may survive for some time period (during which it drives an inflationary expansion) until it eventually "decays", whereas in a model including wormholes it should rapidly fall to zero, once the size of the universe exceeds the wormhole scale [5] (although the details of this process are unknown). Whether the universe will actually undergo an inflationary phase after nucleation will certainly be affected by the answer to these (and related) fundamental questions, as well as by the field content and matter couplings. Moreover, in the case the universe nucleates at a dimension larger than four, and the initial hypersurface has the product structure $\Sigma=\mathcal{N}_{n-4} \times \mathcal{N}_{3}$, it is conceivable that the field couplings give rise to some KaluzaKlein mechanism that captures the factor $\mathcal{M}_{n-4}$ at unobservably small sizes (see e.g. [37] and [6]). In this case, space-time appears to be four-dimensional although at a more fundamental level it is of dimension $n$ (see e.g. Refs. [38] and [39]). If such matter couplings are absent, the primordial ("frozen out") cosmological constant will "decay" or be driven to zero on account of the wormhole mechanism, and eventually all dimensions blow up. We will keep in mind that we cannot exclude any of these possibilities at the principal level of our considerations.

Returning to the technical aspects of our suggestion, we have to note that there is one unknown fundamental quantity remaining, namely the $n$-dimensional gravitational constant $G_{n}$, as showing up in (2.1). We exclude it from effectively depending on other vaiables. (Since in the wormhole-based models it appears to be bounded away from zero anyway, our assumption to treat it as a true constant does not seem to be an oversimplification; cf. Refs. 40] and 41]). From dimensional arguments, we know that $G_{n} \sim m_{P}^{2-n}$, but we do not now anything about the prefactor if $n \neq 4$. 
For later convenience, let us write

$$
G_{n}=\left(\frac{\kappa_{n}}{m_{P}}\right)^{n-2}
$$

where the $\kappa_{n}$ are dimensionless numbers, and $\kappa_{4}=1$. Sometimes all $\kappa_{n}$ are set equal to 1 (e.g. in Ref. [7]). However, this need not be the correct choice. When relative probabilities of configurations in different dimensions are compared, one would have to know these numbers. Put otherwise, one can try to set bounds on them by means of physical predictions concerning our universe. However, it appears natural to assume that the $\kappa_{n}$ are of the order 1, and to check the sensitivity of predictions against small modifications thereof. This is what we will do in Section 6, but we should keep in mind that a full understanding of a genuinely multiple-dimensional theory, and a quantum cosmological explanation why we observe a four-dimensional space-time relies on these numbers as well.

In the remaining Sections we will specialize to a simple model in which our suggestion can be tested. As a model for $I$ (whether it is intepreted as fundamental or effective) we consider pure gravity, matter just being represented effectively by a (non-fixed) cosmological constant $\Lambda$. (This corresponds to Coleman's original scenario [5]). Moreover, we will not consider all solutions to the Euclidean Einstein equations (i.e. all Einstein metrics) admitting a symmetric hypersurface $\Sigma$, but only products of (round) spheres of arbitrary dimension. Since not much is known about the set of all Einstein spaces admitting a symmetric hypersurface (cf. [9]), and in view of the belief that this set is probably quite small [42, the restriction to products of spheres may turn to be a viable approximation of the real situation. Within this framework, we can carry out the analysis of relative probabilities and ask for the favoured dimension and topology of the universe. Due to the product structure of the manifolds we consider, we will obtain the principal possibility to include the question whether our universe is of a Kaluza-Klein type, i.e. with several dimensions compactfied to unobservably small size. We will find more or less definite answers to these questions. However, when dealing with numbers, we should not forget that higher loop corrections as well as sophisticated matter sectors could modify our results. 


\section{Action on products of spheres}

In this Section we consider manifolds which are, topologically, products of spheres, i.e.

$$
\mathcal{M}=\mathbf{S}^{n_{1}} \times \mathbf{S}^{n_{2}} \times \ldots \times \mathbf{S}^{n_{m}}
$$

and denote the total dimension of $\mathcal{M}$ by

$$
n=\sum_{B=1}^{m} n_{B}
$$

$m$ being the number of factors. Any of the spheres $\mathbf{S}^{n_{B}}$ shall carry the standard (round) metric with radius $a_{B}$, which implies that its curvature scalar is given by $R_{B}=n_{B}\left(n_{B}-1\right) / a_{B}^{2}$.

Our next assumption is that the total metric thus defined on $\mathcal{M}$ satisfies the Euclidean Einstein equations (2.2) with cosmological constant $\Lambda>0$ (and $\left.T_{\mu \nu}^{\text {else }}=0\right)$. Here, some trivial cases are possible: If $n=1$ or $n=2$, the Einstein equations lead to $\Lambda=0$. If $n>2$ and at least one of the spheres is one-dimensional, the Einstein equations imply $n_{B}=1$ for all $B=1, \ldots m$, and $\Lambda=0$. We will not consider these geometries, and restrict the sequence of dimensions $\left\{n_{1}, \ldots n_{m}\right\}$ to the generic case $n>2$ and $n_{B}>1$ for all $B$. Hence, the lowest possible dimension is $n=3$, and it is realized only by $\mathcal{M}=\mathbf{S}^{3}$ (this configuration will later on be excluded by a mechanism similar to the one proposed by Gasperini [7]). The next possible dimension, $n=4$, is realized by $\mathcal{M}=\mathbf{S}^{4}$ and $\mathcal{M}=\mathbf{S}^{2} \times \mathbf{S}^{2}$.

In the generic case, as specified above, the Einstein equations are equivalent to the system of equations

$$
\frac{n_{B}-1}{a_{B}^{2}}=\frac{2 \Lambda}{n-2} \quad B=1, \ldots m .
$$

Hence, for any given $\Lambda>0$, the radii of the factor-spheres are uniquely determined to be

$$
a_{B}=\left(\frac{\left(n_{B}-1\right)(n-2)}{2 \Lambda}\right)^{1 / 2} \quad B=1, \ldots m .
$$

Any Einstein space constructed in this way has non-zero curvature, and its volume is given by

$$
\mathcal{V}=\left(\frac{n-2}{2 \Lambda}\right)^{n / 2} \prod_{B=1}^{m} v_{n_{B}}\left(n_{B}-1\right)^{n_{B} / 2}
$$


where

$$
v_{p}=\frac{2 \pi^{(p+1) / 2}}{\Gamma\left(\frac{p+1}{2}\right)}
$$

is the volume of the unit $p$-sphere. The value of the Euclidean action (2.1), when such a solution is inserted, turns out to be (using $R=2 n \Lambda /(n-2)$ for the total curvature scalar, which follows from contracting (2.2))

$$
I=-\frac{\mathcal{V}}{8 \pi G_{n}} \frac{2 \Lambda}{n-2}=-\frac{1}{8 \pi G_{n}}\left(\frac{n-2}{2 \Lambda}\right)^{(n-2) / 2} \prod_{B=1}^{m} v_{n_{B}}\left(n_{B}-1\right)^{n_{B} / 2} .
$$

For given $n$ and $\Lambda$, the minimum Euclidean action is attained for the single-sphere $(m=1)$ configuration $\mathcal{M}=\mathbf{S}^{n}$, and this provides, as already mentioned, a general lower bound for the value of the Euclidean action, evaluated at arbitrary compact solutions of the Euclidean Einstein equations [16]. The variables symbolically denoted by $\gamma$ in Section 2 are now provided by $\left\{n_{1}, \ldots n_{m}\right\}$, and (4.7) is just the expression $I_{\Lambda}(\gamma)$.

Any of our configurations $\mathcal{M}$ carries hypersurfaces $\Sigma$ with zero extrinsic curvature (i.e. symmetric hypersurfaces), at which the universe can nucleate. Since our configurations $\mathcal{M}$ are products of spheres, the possible hypersurfaces $\Sigma$ are provided by the equators of the factor-spheres. Let $\Sigma_{A}$ denote the hypersurface of $\mathcal{M}$ which corresponds to the equator of the $A$-th sphere $\mathbf{S}^{n_{A}}$ (it is defined as the set of all points $\left(x_{1}, \ldots, x_{A}, \ldots, x_{m}\right)$, where $x_{A}$ is on the equator of the $A$-th sphere, while all other $x_{B}$ are points on the $B$-th sphere without further restriction). This means that we assume a situation analogous to (2.3) in the $A$-th factor-sphere. In terms of classical signature change, one $\mathbf{S}^{n_{A}}$ induces the emergence of a Lorentzian time coordinate and a subsequent classical evolution of the corresponding spacelike slices (which are topologically products of $\mathbf{S}^{n_{A}-1}$ with the remaining unaffected spheres). If we assume $\Lambda$ to maintain its (non-zero) value after nucleation (and henceforth be a constant), the $\mathbf{S}^{n_{A}}$-part of $\Sigma_{A}$ will inflate while all other factor-spheres remain "small". (In the language of Kaluza-Klein theories, one would state that they remain "compactified"). However, since $\Lambda$ is only an effective phenomenon, it might rapidly decay as well, the details depending on the particular theory that is approximated by our simplified model, and we shall not specify them.

Thus, assuming the $A$-th sphere of $\mathcal{M}$ to induce a change of metric signature, we arrive at a "real tunneling geometry"

$$
\mathcal{M}_{\text {real tunneling }}=\mathbf{S}^{n_{1}} \times \ldots \times \mathbf{K}^{n_{A}} \times \ldots \times \mathbf{S}^{n_{m}}
$$


The Euclidean part of this space is just the half of (4.1), and the matching to the classical Lorentzian universe is along $\Sigma_{A}$.

Having specified the family of pairs $(\mathcal{M}, \Sigma)$ that may occur in this simplified version of our proposal, we begin carrying out the analysis described in the foregoing Section by computing the nucleation energy $E$. Once $(\mathcal{M}, \Sigma)$ (i.e. $\left\{n_{1}, \ldots n_{m}\right\}$ and $A)$ are fixed, the effective non-gravitational energy generated at $\Sigma_{A}$ is given by

$$
E=\frac{1}{8 \pi G_{n}} \mathcal{V}_{\Sigma_{A}} \Lambda
$$

where

$$
\mathcal{V}_{\Sigma_{A}}=\frac{v_{n_{A}-1}}{v_{n_{A}} a_{A}} \mathcal{V}=\left(\frac{n-2}{2 \Lambda}\right)^{(n-1) / 2} \frac{v_{n_{A}-1}}{v_{n_{A}}\left(n_{A}-1\right)^{1 / 2}} \prod_{B=1}^{m} v_{n_{B}}\left(n_{B}-1\right)^{n_{B} / 2}
$$

is the volume of $\Sigma_{A}$ as an $\left(n_{A}-1\right)$-manifold. As a consequence,

$$
E=\frac{1}{8 \pi G_{n}} \frac{v_{n_{A}-1}}{v_{n_{A}} a_{A}} \mathcal{V} \Lambda=-\frac{v_{n_{A}-1}(n-2)}{2 v_{n_{A}} a_{A}} I
$$

When expressed entirely in terms of $\Lambda$ (using (4.4) and (4.5)), we obtain

$$
E=\frac{1}{16 \pi G_{n}} \frac{v_{n_{A}-1}(n-2)}{v_{n_{A}}\left(n_{A}-1\right)^{1 / 2}}\left(\frac{n-2}{2 \Lambda}\right)^{(n-3) / 2} \prod_{B=1}^{m} v_{n_{B}}\left(n_{B}-1\right)^{n_{B} / 2} .
$$

For any given configuration (with $n \neq 3$ ) this provides a one-to-one correspondence between the cosmological constant and the nucleation energy. The set of variables denoted by $\alpha$ in the preceding Sections is now given by $\left\{n_{1}, \ldots n_{m}\right\}$ together with $A$. The Euclidean action may thus likewise be expressed in terms of the variables $(\Lambda, \gamma)$ or $(E, \alpha)$.

We note that for $n=3$, the energy is independent of $\Lambda$, and is given by $E=1 /\left(6 G_{3}\right)$. Hence, if $E$ plays the role of a quantity whose value is generic and only dynamically governed by a probability law, it appears unlikely that the $n=3$ case will contribute to the fluctuations between (or succession of) our various configurations $\mathcal{M}$. Hence, in this sence, the minimum dimensionality of space-time is predicted to be 4. This is quite similar to the reasoning of Gasperini [7].

If $n>3$, the relation (4.12) can be used to express $\Lambda$ in terms of $E$. Inserting the result into (4.7), we find the action expressed entirely in terms of the energy 
$E$, the dimensions $\left\{n_{1}, \ldots, n_{m}\right\}$ (characterizing the topology of $\mathcal{M}$ ) and the number $A$ of the sphere which induces the transition to a classical universe. Using (3.3) in addition, we arrive at the expression

$$
I=-\left(\frac{2 \kappa_{n}}{n-2} \frac{E}{m_{P}} \frac{v_{n_{A}}\left(n_{A}-1\right)^{1 / 2}}{v_{n_{A}-1}}\right)^{\frac{n-2}{n-3}}\left(\frac{1}{8 \pi} \prod_{B=1}^{m} v_{n_{B}}\left(n_{B}-1\right)^{n_{B} / 2}\right)^{-\frac{1}{n-3}}
$$

which corresponds to what was denoted by $I_{E}(\alpha)$ in Section 3. It is the starting point for our quantitative analysis.

\section{Favoured topologies at fixed dimension}

According to our proposal, the first step is to analyze the values of the action for constant $E$. Here we encounter the fact that $I$ from (4.13) contains the unkown numbers $\kappa_{n}$. However, if $n$ is fixed in addition, we can at least distinguish between different topologies. Hence, let us postpone the question which dimension minimizes $I$ at a given value of $E$ to the next Section, and suppose $n$ to be fixed as well as $E$. Thus the question which topology is favoured if the universe nucleates as an $n$-dimensional space-time becomes a well-posed problem.

In order to get a more handsome quantity, we omit the irrelevant factors in $I$ containing only $n$ and $E$, and raise to the power $3-n$ (which is assumed to be negative from now on). The most probable configuration is the one minimizing

$$
F=\left(\frac{v_{n_{A}-1}}{v_{n_{A}}\left(n_{A}-1\right)^{1 / 2}}\right)^{n-2} \prod_{B=1}^{m} v_{n_{B}}\left(n_{B}-1\right)^{n_{B} / 2} .
$$

The complete Euclidean action is given by

$$
I=-\left(\frac{8 \pi}{F}\right)^{1 /(n-3)}\left(\frac{2 \kappa_{n}}{n-2} \frac{E}{m_{P}}\right)^{(n-2) /(n-3)} .
$$

Minimizing $F$ is easily done for small values of $n$ by explicitly inserting the candidate configurations. In order to have a convenient notation, we will denote the $A$-th sphere (the one that undergoes a signature change) by a tilde, hence write

$$
\mathcal{M}=\mathbf{S}^{n_{1}} \times \ldots \times \widetilde{\mathbf{S}^{n_{A}}} \times \ldots \mathbf{S}^{n_{m}}
$$


For $n=4$ and $n=5$ we find

$$
\begin{aligned}
F\left(\widetilde{\mathbf{S}^{4}}\right) & =\frac{9 \pi^{2}}{2} \approx 44.4132 \\
F\left(\mathbf{S}^{2} \times \widetilde{\mathbf{S}^{2}}\right) & =4 \pi^{2} \approx 39.4784 \\
F\left(\widetilde{\mathbf{S}^{5}}\right) & =\frac{2048}{27} \approx 75.8519 \\
F\left(\widetilde{\mathbf{S}^{2}} \times \mathbf{S}^{3}\right) & =2 \sqrt{2} \pi^{3} \approx 87.699 \\
F\left(\mathbf{S}^{2} \times \widetilde{\mathbf{S}^{3}}\right) & =64 .
\end{aligned}
$$

Hence, in 4 dimensions, $\mathbf{S}^{2} \times \widetilde{\mathbf{S}^{2}}$ is favoured over $\widetilde{\mathbf{S}^{4}}$, and in 5 dimensions $\mathbf{S}^{2} \times \widetilde{\mathbf{S}^{3}}$ is the winner. Let us write down a list of the configurations $\mathcal{K}_{n}$ minimizing $F$ for $n=4, \ldots 15$

$$
\begin{aligned}
\mathcal{K}_{4} & =\mathbf{S}^{2} \times \widetilde{\mathbf{S}^{2}} \\
\mathcal{K}_{5} & =\mathbf{S}^{2} \times \widetilde{\mathbf{S}^{3}} \\
\mathcal{K}_{6} & =\mathbf{S}^{2} \times \widetilde{\mathbf{S}^{4}} \\
\mathcal{K}_{7} & =\mathbf{S}^{2} \times \mathbf{S}^{2} \times \widetilde{\mathbf{S}^{3}} \\
\mathcal{K}_{8} & =\mathbf{S}^{2} \times \mathbf{S}^{2} \times \widetilde{\mathbf{S}^{4}} \\
\mathcal{K}_{9} & =\mathbf{S}^{2} \times \mathbf{S}^{2} \times \widetilde{\mathbf{S}^{5}} \\
\mathcal{K}_{10} & =\mathbf{S}^{2} \times \mathbf{S}^{2} \times \mathbf{S}^{2} \times \widetilde{\mathbf{S}^{4}} \\
\mathcal{K}_{11} & =\mathbf{S}^{2} \times \mathbf{S}^{2} \times \mathbf{S}^{2} \times \widetilde{\mathbf{S}^{5}} \\
\mathcal{K}_{12} & =\mathbf{S}^{2} \times \mathbf{S}^{2} \times \mathbf{S}^{2} \times \mathbf{S}^{2} \times \widetilde{\mathbf{S}^{4}} \\
\mathcal{K}_{13} & =\mathbf{S}^{2} \times \mathbf{S}^{2} \times \mathbf{S}^{2} \times \mathbf{S}^{2} \times \widetilde{\mathbf{S}^{5}} \\
\mathcal{K}_{14} & =\mathbf{S}^{2} \times \mathbf{S}^{2} \times \mathbf{S}^{2} \times \mathbf{S}^{2} \times \widetilde{\mathbf{S}^{6}} \\
\mathcal{K}_{15} & =\mathbf{S}^{2} \times \mathbf{S}^{2} \times \mathbf{S}^{2} \times \mathbf{S}^{2} \times \mathbf{S}^{2} \times \widetilde{\mathbf{S}^{5}}
\end{aligned}
$$

Hence, there is a clear tendency for $F$ to be minimized by products of several $\mathbf{S}^{2}$ with a higher dimensional $\mathbf{S}^{p}$. Moreover, in all configurations, the signature changing sphere is the one with the highest dimension. This is valid for all $n$ (it is a trivial consequence of the numerics of $\left.v_{p}\right)$. For the dimensions $(16, \ldots 42)$ the according configurations are products of two-spheres with an $\widetilde{\mathbf{S}^{p}}$, where $p$ takes the values $(6,5,6,7,6,7,6,7,6,7,8,7,8,7,8,7,8,9,8,9,8,9,8,9,8,9,10)$. Hence, the increase of $p$ with $n$ is rather modest, and $n=12$ is the highest dimension in which a factor sphere $\mathbf{S}^{4}$ exists. 
Let us analyze what happens asymptotically for large $n$. The configuration minimizing $F$ is a $q$-fold product of two-spheres with an $\widetilde{\mathbf{S}^{p}}$, hence $m=q+1$ and $2 q+p=n$. Evaluated at these numbers, $F$ becomes

$$
F=(4 \pi)^{(n-p) / 2}\left(\frac{v_{p-1}}{v_{p}(p-1)^{1 / 2}}\right)^{n-2} v_{p}(p-1)^{p / 2} .
$$

Treating $p$ as a continuous variable, this expression can be differentiated with respect to $p$. One thereby encounters the so-called logarithmic derivative of the Gammafunction whose expansion for large arguments is

$$
\Omega(x) \equiv \frac{\Gamma^{\prime}[x]}{\Gamma[x]}=-\ln \left(\frac{1}{x}\right)-\frac{1}{2 x}-\frac{1}{12 x^{2}}+O\left(\frac{1}{x^{4}}\right) .
$$

Since $\partial F / \partial p=0$ reduces to an expression of first order in $n$, it can be solved to give

$$
n=\frac{p+2+(p-1)\left(\ln (p-1)-2 \ln 2+2 \Omega\left(\frac{p}{2}\right)-3 \Omega\left(\frac{p+1}{2}\right)\right)}{1+(p-1)\left(\Omega\left(\frac{p}{2}\right)-\Omega\left(\frac{p+1}{2}\right)\right)} .
$$

Insertion of the asymptotic behaviour (5.22) yields

$$
n=(1-\ln 2)\left(2 p^{2}-4 p\right)+\frac{17}{3}-3 \ln 2+O\left(\frac{1}{p^{2}}\right) .
$$

Inverting the series, and neglecting contributions which vanish as $n \rightarrow \infty$, we find the dimension of the signature changing sphere in $\mathcal{K}_{n}$ for large $n$ to be

$$
p \approx \beta \sqrt{n}+1
$$

with $\beta=(2(1-\ln 2))^{-1 / 2} \approx 1.2765$. This explains the slow increase of the factor spheres' dimensions we observed before. The error in (5.25) will, by the way, be of order 1 even for $n \rightarrow \infty$, because both $p$ and $n$ are integers (a fact that is ignored by the continuous approximation).

In order to know the approximate value of $F$ at these configurations, we use Stirling's formula for the Gamma-function to obtain after a lengthy computation

$$
F\left(\mathcal{K}_{n}\right)=2^{(n+2) / 2} \pi \exp \left(\frac{\sqrt{n}}{2 \beta}\right)\left(1+O\left(\frac{1}{\sqrt{n}}\right)\right) .
$$


For the low dimensions this formula is of course not very accurate but displays the qualitative behaviour quite well. If $n$ varies from 4 to 15 (corresponding to the list (5.9)-(5.20), the relative deviation from the exact value is between $12 \%$ and $29 \%$.

For comparison we also write down a similar expansion for $F$ evaluated on singlesphere $(m=1)$ configurations with large dimension,

$$
F\left(\mathbf{S}^{n}\right)=2^{3 / 2} \pi \exp \left(\frac{n}{2}-\frac{1}{4}\right)\left(1+O\left(\frac{1}{n}\right)\right) .
$$

The dominant contributions to the exponential increase

$$
F\left(\mathcal{K}_{n}\right) \sim 2^{n / 2}, \quad F\left(\mathbf{S}^{n}\right) \sim e^{n / 2}
$$

show how the products $\mathcal{K}_{n}$ are favoured over the single-spheres $\mathbf{S}^{n}$.

Do these results already give rise to predictions? We will confirm in Section 7 that - as a consequence of our proposal - the small dimensions are favoured over the large ones when the universe nucleates. Thus, if the minimization of $I$ is applied straightforwardly, we would predict $n=4$ and the global topology of the spatial sections of the universe to be the same as the signature changing hypersurface $\Sigma$ of $\mathcal{K}_{4}$, hence $\mathbf{S}^{1} \times \mathbf{S}^{2}$. In other words, the universe is of Kantowski-Sachs type, a result that might be not very appealing (although consistent with observations). However, we must keep in mind that we have not inserted a particular model for the matter sector (except for an effective cosmological constant). This leaves some freedom for an alternative scenario: As we mentioned in Section 3 the mechanism selecting a classical universe might pick a configuration with large (but possibly not the largest) probability. In other words, it might drive the universe classical "before" the absolute minimum of $n$ is reached. Furthermore, the wormhole scale (which we do not know) provides some uncertainty about these things. Hence, if the selected configuration is not $\mathcal{K}_{4}$ but some other $\mathcal{K}_{n}$ (with small $n$, which we may reasonably assume), and if its nucleation is accompagnied with a subsequent Kaluza-Klein mechanism [38 that captures some of the scale factors, we might end up with a space-time containing unobservably small internal spaces. The most appealing candidates in our list (5.9)-(5.20) of minimizing configurations $\mathcal{K}_{n}$ are of course those containing a factor $\widetilde{\mathbf{S}^{4}}$. One of the four dimensions is absorbed by the emergence of Lorentzian time, and the according equator $\mathbf{S}^{3}$ represents the observable space-like slices of our universe. This mechanism is possible in $n=6,8$, 10 and 12 dimensions, the internal spaces being products of two-spheres. 
We should add that including a more sophisticated matter sector in the action I could substantially modify these results (cf. Ref. [37] for supergavity, Ref. [6] for six-dimensional Einstein-Maxwell theory and Ref. 8] for an axion field), and it is presumably easily to find models in which $\Sigma=\mathbf{S}^{3}$ is the most probable initial slice, even without Kaluza-Klein effect. On the other hand, if the possibility of wormholes is admitted, and the non-gravitational degrees of freedom do not give rise to vacuum energies other than those represented by $\Lambda$, the model considered here should apply quite well. In the case such a version of a wormhole-based model applies for energies $E$ substantially larger than the wormhole scale, the prediction for the spatial slices is $\mathbf{S}^{1} \times \mathbf{S}^{2}$.

Thus, even the first step in our procedure (together with a slight anticipation of the following) has revealed non-trivial statements about the global topology of space-time. The next - somewhat intermediate - step will be to examine the value of the action at constant $E$ but variable $n$.

\section{Favoured dimension at fixed energy}

Turning to the question which dimension is favoured at a given nucleation energy $E$, we run inevitably into the problem that the numbers $\kappa_{n}$ in the $n$-dimensional gravitational constant are unknown (except for $\kappa_{4}=1$ ). In lack of a better knowledge about this, we will base our arguments on the expectation that $\kappa_{n}$ does not deviate much from unity. Let us begin however leaving $\kappa_{n}$ unconstrained.

The results of the foregoing Section amount to associate with each value of $n$ a Euclidean configuration $\mathcal{K}_{n}$, minimizing the quantity $F$ from (5.1). Thus the question for the favoured dimension is traced back to the minimization of $I_{E}\left(\mathcal{K}_{n}\right)$ at constant $E$. Using the form (5.2) and the asymptotic behaviour (5.26), we note that $F\left(\mathcal{K}_{n}\right)^{-1 /(n-3)} \rightarrow 1 / \sqrt{2}$ as $n \rightarrow \infty$, and

$$
I_{E}\left(\mathcal{K}_{n}\right)=-\sqrt{2}\left(\frac{\kappa_{n}}{n-2}\right)^{(n-2) /(n-3)} \frac{E}{m_{P}}\left(1+O\left(\frac{1}{n}\right)\right) .
$$

This shows the extent to which the whole procedure is sensitive to the values of $\kappa_{n}$. If $\kappa_{n} / n \rightarrow 0$ as $n \rightarrow \infty$, the action will approach zero from below, and will definitely admit a finite value of $n$ where it attains its minimum. If $\kappa_{n} / n$ blows up, $I$ will tend towards $-\infty$, thus having no minimum. In what follows we assume the former case to be true. (Let us just mention that in the case $\kappa_{n} / n$ blows up as $n \rightarrow \infty$, the 
action will attain a maximum at some finite $n$. Such a modification could account for the reversed sign the action enters the tunneling proposal approach by Vilenkin and Linde [31, [34], 36], where $P \sim \exp (I))$.

Let us return to the exact expressions and ask what happens if $E$ is very large. The relevant term in $I$ is then provided by $\left(E / m_{P}\right)^{(n-2) /(n-3)}$, which is, for $E>m_{P}$, a monotonically decreasing function in $n$ that approaches 1 at infinity. As long as $\kappa_{n} / n$ decreases to zero for large $n$, the minimizing dimension can be made smaller by increasing $E$. Hence, if $E \gg m_{P}$, the action is minimized at the lowest possible dimension, $n=4$. For such a large value of $E$ one has, clearly, $I_{E}\left(\mathcal{K}_{4}\right)>I_{E}\left(\mathcal{K}_{n}\right)$ for all $n \geq 5$. Decreasing $E$, things can change only if at some threshold $E=E_{4}$, the action of $\mathcal{K}_{4}$ becomes equal to the action of some higher $\mathcal{K}_{n}$. Note that, from the outset, it is not at all clear whether such a threshold would occur at $n=5$ or at some higher dimension. Again, this depends on the constants $\kappa_{n}$ (and in particular on their values for small $n$ ).

A selection of energy values defined by such identities is

$$
\begin{aligned}
I_{E}\left(\mathcal{K}_{4}\right)=I_{E}\left(\mathcal{K}_{5}\right) & \text { at } & E & =\frac{\pi^{3}}{108} \kappa_{5}^{3} \kappa_{4}^{-4} m_{P}, \\
I_{E}\left(\mathcal{K}_{4}\right)=I_{E}\left(\mathcal{K}_{6}\right) & \text { at } & E & =\frac{\sqrt{\pi}}{3 \sqrt{6}} \kappa_{6}^{2} \kappa_{4}^{-3} m_{P}, \\
I_{E}\left(\mathcal{K}_{5}\right)=I_{E}\left(\mathcal{K}_{6}\right) & \text { at } & E & =\frac{432}{\pi^{7}} \kappa_{6}^{8} \kappa_{5}^{-9} m_{P} .
\end{aligned}
$$

This illustrates that one can easily create various different situations by accordingly adjusting the $\kappa_{n}$. This is of course not what we are interested it. A reasonable condition on the $\kappa_{n}$ is certainly provided by some sort of monotonicity. The result will in general be a monotonic order of the favoured dimension as $E$ decreases. In order not to overcomplicate things, let us from now on make the usual choice

$$
\kappa_{n}=1 \quad \text { for all } n \text {. }
$$

Small modifications of these numbers will not change any of our principal conclusions. (Recall that the prediction of $\mathcal{K}_{4}$ as the nucleation geometry at scales $E \gg m_{P}$ is not affected thereof.)

Having fixed the remaining freedom, the further analysis is straightforward. The first "transition" occurs at $I_{E}\left(\mathcal{K}_{4}\right)=I_{E}\left(\mathcal{K}_{5}\right)$, hence

$$
E_{4}=2^{-2} 3^{-3} \pi^{3} m_{P} \approx 0.2871 m_{P}
$$


Let us define in general $E_{n}$ by $\mathcal{K}_{n}$ and $\mathcal{K}_{n+1}$ having equal action. We easily find the first few values

$$
\begin{aligned}
& E_{5}=2^{4} 3^{3} \pi^{-7} m_{P} \approx 0.1430 m_{P}, \\
& E_{6}=3^{12} 2^{-11} 5^{-15} \pi^{14} m_{P} \approx 0.07757 m_{P}, \\
& E_{7}=2^{37} 5^{25} 3^{-40} \pi^{-22} m_{P} \approx 0.03892 m_{P} .
\end{aligned}
$$

Thus we encounter a decreasing sequence of energy scales. For $E>E_{4}$ the action is minimized by $\mathcal{K}_{4}$, hence the dimension 4 . If $E_{n-1}>E>E_{n}$, the favoured configuration is $\mathcal{K}_{n}$, the according dimension is $n$.

The asymptotic regime of small $E$ is governed by the expansion (5.26). Again, an awkward computations, which needs the expansion

$$
\frac{F\left(\mathcal{K}_{n}\right)}{F\left(\mathcal{K}_{n+1}\right)}=\frac{1}{\sqrt{2}} \exp \left(-\frac{1}{4 \beta \sqrt{n}}\right)\left(1+O\left(\frac{1}{n^{3 / 2}}\right)\right)
$$

as as intermediate step, reveals for large $n$

$$
\frac{E_{n}}{m_{P}}=\frac{n}{2^{3 / 2}} \exp \left(-n+\frac{\sqrt{n}}{4 \beta}+\frac{5}{2}\right)\left(1+O\left(\frac{1}{\sqrt{n}}\right)\right) .
$$

Note that the level spacing $\Delta E \equiv E_{n-1}-E_{n}$ is of the order of $E$ itself, $\Delta E \approx E$. Inverting this expression up to the first two orders, it follows that the favoured dimension for $E \ll m_{P}$ is

$$
n \approx \ln \left(\frac{m_{P}}{E}\right)+\frac{1}{4 \beta}\left(\ln \left(\frac{m_{P}}{E}\right)\right)^{1 / 2} .
$$

Although this formula is probably going to be modified by higher loop corrections, it nicely display what one means by a higher-dimensional phase in the early universe. In absence of a decoherence mechanism as very small energies, one could even say that all of these dimensions contribute and talk about a multiple-dimensional state. This can be viewed as a quantum version of the idea that the universe "begins" as a cold and small (though higher-dimensional) one. (The nucleation volume is in fact very small for large $n$, whereas the nucleation radius is large, see equations (6.13) and (6.14) below).

As an alternative method, one could have improved the expansion (5.26) by including the next order, and differentated $I$ directly with respect to $n$ in order to 
find the minimizing dimension. In order to further characterize the configurations $\mathcal{K}_{n}$, one may derive asymptotic expressions for their total action, the nucleation volume $\mathcal{V}_{\Sigma} \equiv \mathcal{V}_{A}$ and the total $n$-volume $\mathcal{V}$, as well as for the radius $a_{A}$ of the nucleating factor $\widetilde{\mathbf{S}^{p}}$ and the according value of the cosmological constant $\Lambda$. In order not to present too much technical details, we just write down qualitatively the leading behaviour for large $n$ (small $E$ )

$$
\begin{aligned}
-I & \sim \mathcal{V}_{A} \sim \mathcal{V} \sim e^{-n} \sim E \\
a_{A} & \sim n^{1 / 4} \sim(-\ln E)^{1 / 4} \\
\Lambda & \sim n \sim-\ln E .
\end{aligned}
$$

Note that due to the bunch of $\mathbf{S}^{2}$-factor-spheres in the higher $\mathcal{K}_{n}$, the relation between the cosmological constant and the nucleation radius is not the standard one $\left(\Lambda \sim a_{A}^{4}\right.$ instead of $\Lambda \sim a_{A}{ }^{-2}$, see also equation (4.4)). On the other side of the spectrum, for $E>E_{4}$, the favoured configuration is $\mathcal{K}_{4}$, which implies

$$
\Lambda=\pi^{2}\left(\frac{m_{P}}{E}\right)^{2} m_{P}^{2}=a_{A}{ }^{-2} .
$$

\section{$7 \quad$ Favoured energy}

Having exhibited the favoured configurations at fixed $E$, the last step is to evaluate the values of the action among these. (Asymptotically for small $E$, we have done this already in the preceding Section, see (6.1) and (6.12) $)$. One might try to relate this step to the question for the most probable energy the actual classical universe will start with. However, the answer will be $E=\infty$, and, as already stated, we prefer to talk about some mechanism that will drive the universe to become classical at a finite value of $E$. In Section 6 we found a threshold $E_{4}$ above which the variables $\left\{n_{1}, \ldots n_{m}\right\}$ and $A$ have settled and remain constant. This threshold is of course a natural candidate for the transition to a real universe. However, since we neither understand the details of this process nor have any knowledge of the wormhole scale $E_{\text {wh }}$, it is conceivable that nucleation occurs only approximately at $E_{4}$. In the case of $E<E_{4}$, it may not be the true minimizing configuration $\Sigma=\mathbf{S}^{1} \times \mathbf{S}^{2}$ (from $\mathcal{K}_{4}$ ) that is "frozen out" but one that can be expected to be among the next few on the list (5.9)-(5.20). As already mentioned in Section 5, this provides the possibility for a Kaluza-Klein scenario. 
Since the minimizing configurations at fixed $E$ (i.e. the functions $\alpha(E)$ in the notation of Section 3) are already determined, there remains rather little work to do. Let us denote by $I(E)$ the action at the favoured configuration associated with the energy $E$, i.e. the quantity we have called $I_{E}(\alpha(E))$ symbolically. For small $E$, (6.1) and (6.12) can be combined to give, to leading order,

$$
I(E) \approx-\sqrt{2}\left(\ln \left(\frac{m_{P}}{E}\right)\right)^{-1} \frac{E}{m_{P}}
$$

which implies $\ln (-I) \sim \ln (E)-\ln (-\ln (E))$, hence a behaviour quite close to linear $(I \sim-E)$, and in particular $I(0)=0$. Inside any interval $E_{n-1}<E<E_{n}$ the favoured dimension is $n$, and the action is given by (5.2), i.e.

$$
I(E)=-k_{n} E^{(n-2) /(n-3)}
$$

where $k_{n}$ is a positive constant. This is a monotonically decreasing function. Furthermore, from Section 6 we know that $I(E)$ is continuous, because is has equal value for both adjacent configurations at the boundaries $E_{n}$ of the intervals. Finally, for $E>E_{4}$, we have dimension 4 and $I(E)=-k E^{2}$. To summarize, $I(E)$ is a continuous, monotonically decreasing function, thus favours large $E$. Thereby, the formal result of minimizing the action, $E \rightarrow \infty$, is technically analogous to $\Lambda \downarrow 0$ in Coleman's theory.

This is the final (although heuristic) step in our argumentation: The relative order that is induced by the action on the set of $\mathcal{K}_{n}$ is from small to large energies, hence from high to low dimensions. At $E \approx E_{4}$, the large-scale variables (like dimension and topology) become classical (whereas the small-scale degrees of freedom might continue to be quantum mechanical). Hence, the energy scale $E \approx E_{4}$ is related to the phase of nucleation, while the scale $E \ll E_{4}$ corresponds to the genuine multiple-dimensional quantum state.

In the case our model is intended to represent a wormhole-based scenario, one might ask whether the framework we have developed is of some significance for the late universe too. For $E>E_{4}$, the minimizing configuration is $\mathcal{K}_{4}$. Due to (6.16), $\Lambda$ is predicted to approach zero as $E$ blows up. The effective action for the late universe (as long as $\mathcal{K}_{4}$ is actually selected to become real) is given by

$$
I\left(\mathcal{K}_{4}\right)=-\frac{2}{\pi}\left(\frac{E}{m_{P}}\right)^{2}=-2 \pi \frac{m_{P}^{2}}{\Lambda} .
$$


It can formally be inserted into Coleman's measure for the wormhole parameters (i.e. the variables $\Lambda$ depends on). Since it differs from $I\left(\mathbf{S}^{4}\right)$ only by a constant prefactor, the familiar $\delta(\Lambda)$-peak is produced. In terms of the energy, this would result into the (naive) prediction that $E$ is not just very large but actually infinite. A possible implementation of our suggestion in Coleman's model could be to consider $E$ as a function of the wormhole parameters, and to treat the appearance of arbitrarily large universes not just by a cutoff that is sent to infinity [5], but by some cosmologically more appealing regularization method. Thus it would be a major challenge to modify or rephrase the wormhole formalism such that is can give rise to sensible predictions for global cosmological variables (as e.g. $E$, if it can be interpreted as the total matter energy contained in the universe, and $\Sigma$, which may represent its size as well as its large-scale topology). The goal of such a theory would be to give statements like $E \rightarrow \infty$ a significance as precise as the famous " $\Lambda=0$ " in Coleman's work. One possible question is whether the "nucleation picture" becomes meaningless after nucleation (i.e. whether the large universe may be understood in terms of "competing" tunneling configurations as well). The cosmological constant might in such a framework just be a secondary quantity (the density associated with $E$ and $\Sigma$, or, more general, the constant $\Lambda$ appearing in (3.2)). The most reasonable point to make contact between our framework and the standard wormhole theory is provided by equation (7.3). If the details of the decoherence mechanism or some non-trivial matter sector induce nucleation around some other configuration than $\mathcal{K}_{4}$, a modified action $I \sim-E^{(n-2) /(n-3)} \sim-\Lambda^{-(n-2) / 2}$ takes over the role of $(7.3)$.

\section{Speculations}

We would like to finish this paper by two comments. The first one is devoted to the idea that one could try to modify the gravitational action by adding an appropriate integral over $\partial \mathcal{M}$ to (2.1), as for example

$$
m_{P}^{n-3} \int_{\partial \mathcal{M}} d^{n-1} x \sqrt{h}\left(c_{1} K^{i j} K_{i j}+c_{2} K^{2}\right) .
$$

Such terms might effectively be generated by higher loop or higher order curvature contributions (see Refs. [43]- [44] for boundary terms in higher derivative gravity). This can lead to a model in which the stationary points of the action are only those solutions of (2.2) for which the boundary data are time-symmetric (which implies in the gravitational sector that the extrinsic curvature $K_{i j}$ vanishes). Given a manifold 
$\mathcal{N}$ with data $(h, \phi)$ that are supposed to match a full Euclidean solution $(\mathcal{M}, g, \Phi)$ at $\partial \mathcal{M}=\mathcal{N}$ (as is required in the Hartle-Hawking formalism), the immediate consequence of $\delta I=0$ is that $(\mathcal{M}, g, \Phi)$ has to be a real tunneling configuration with $\Sigma=\mathcal{N}$. Thus it is possible that for certain $(\mathcal{N}, h, \phi)$ (which are the arguments of the wave function $\psi$ ) there is no stationary point at all. In the case the data on $\mathcal{N}$ do admit a properly matching Euclidean solution, the dominant contribution in the path integral is given by $\exp (-I / 2)$ with $I$ the instanton action. This precisely reproduces the nucleation amplitudes of the Hartle-Hawking formalism. If, on the other hand, the data on $\mathcal{N}$ do not admit a properly matching Euclidean solution, the path integral aquires contributions from various non-stationary configurations, and are thus (leaving aside questions of convergence) expected to be suppressed. A peculiarity of such a formalism is that the wave function does not necessarily oscillate when the scale factors become large. (However, this need not really be a drawback in an effective formalism). In such a model it is not necessary to "associate" the symmetric hypersurfaces $\Sigma$ with the instantons by hand, because the real tunneling configurations play the role of dominant contributions at a fundamental level.

Our last comments concerns the approach suggested by Gasperini [7], that the energy $U$ contained in the Euclidean $n$-geometry of an instanton is conserved under dimensional transitions. As already mentioned in Section 2, it is not clear how $U$ relates to an observed energy. Moreover, Gasperini's framework involves only spheres, and it is not obvious whether it can be generalized to instantons or real tunneling configurations with arbitrary topology. However, one may carry over his idea to the simplified model we considered in Sections $4-7$, by assuming that his definition of $U$ applies for the $A$-th factor sphere, the remaining spheres contributing their full volume, just as contained in $\mathcal{V}_{\Sigma}$. This means that (maybe contrary to the original spirit of Gasperini's work) we use pairs $(\mathcal{M}, \Sigma)$ in order to define $U$. Thus, rescaling $\widetilde{E}=2 \pi U$, we set

$$
\widetilde{E}=\frac{1}{8 \pi G_{n}} \frac{1}{a_{A}} \mathcal{V} \Lambda=-\frac{n-2}{2 a_{A}} I,
$$

hence

$$
\widetilde{E}=\frac{1}{16 \pi G_{n}} \frac{n-2}{\left(n_{A}-1\right)^{1 / 2}}\left(\frac{n-2}{2 \Lambda}\right)^{(n-3) / 2} \prod_{B=1}^{m} v_{n_{B}}\left(n_{B}-1\right)^{n_{B} / 2} .
$$

As a consequence, the Euclidean action may be written as

$$
I=-\left(\frac{2 \kappa_{n}}{n-2} \frac{\widetilde{E}}{m_{P}}\left(n_{A}-1\right)^{1 / 2}\right)^{\frac{n-2}{n-3}}\left(\frac{1}{8 \pi} \prod_{B=1}^{m} v_{n_{B}}\left(n_{B}-1\right)^{n_{B} / 2}\right)^{-\frac{1}{n-3}} .
$$


The according minimization procedure is to look for minimizing configurations at fixed $\widetilde{E}$. The analysis is very similar to the one we have performed in Sections $4-$ 7, the essential difference being that (8.4) favours the single-sphere configurations $\mathbf{S}^{n}$ over the products of spheres (hence over $\mathcal{K}_{n}$ as well). This is a particularly nice feature (it formally predicts not only $n=4$ as the most probable dimension, but also the topology of classical space-time to be $\mathbf{R} \times \mathbf{S}^{3}$ ). The main disadvantage of this approach is in our opinion - apart from the questionable status of $\widetilde{E}$ as observable quantity - that it is not clear how it may be generalized to arbitrary real tunneling configurations. However, it may be considered as an alternative attempt, sharing many technicalities with the one based on the nucleation energy $E$.

\section{References}

[1] J. B. Hartle and S. W. Hawking, "Wave function of the universe", Phys. Rev. D 28, 2960 (1983).

[2] S. W. Hawking, "The quantum state of the universe", Nucl. Phys. B 239, 257 (1984).

[3] S. Giddings and A. Strominger, "Loss of Incoherence and Determination of Coupling Constants in Quantum Gravity", Nucl. Phys. B 307, 854 (1988).

[4] S. Coleman, "Black Holes as Red Herrings: Topological Fluctuations and the Loss of Quantum Coherence", Nucl. Phys. B 307, 867 (1988).

[5] S. Coleman, "Why there is nothing rather than something: A theory of the cosmological constant", Nucl. Phys. B 310, 643 (1988).

[6] I. V. Volovich, "Baby universes and the dimensionality of spacetime", Phys. Lett. $B$ 219, 66 (1989).

[7] M. Gasperini, "The cosmological constant and the dimensionality of spacetime", Phys. Lett. B 224, 49 (1989).

[8] R. C. Myers, "Baby universes in higher dimensional theories", Nucl. Phys. B 323, 225 (1989). 
[9] G. W. Gibbons and J. B. Hartle, "Real tunneling geometries and the large-scale topology of the universe", Phys. Rev. D 42, 2458 (1990).

[10] M. Gell-Mann and J. B. Hartle, "Quantum cosmology and quantum mechanics", in: W. H. Zurek (ed.), Complexity, Entropy and the Physics of Information, Santa Fe Institute Studies in the Sciences of Complexity, vol IX (Addison Wesley, 1990); also in: S. Kobayashi et al (eds.) Proceedings of the 3rd International Symposium on the Foundations of Quantum Mechanics in the Light of New Technology, Physical Society of Japan (Tokyo).

[11] J. B. Hartle, "The quantum mechanics of cosmology", in: S. Coleman et al (eds.), Quantum cosmology and baby universes, World Scientific (Singapore, 1991).

[12] S. W. Hawking, "Quantum gravity and path integrals", Phys. Rev. D 18, 1747 (1978).

[13] G. W. Gibbons, S. W. Hawking and M. J. Perry, "Path integrals and the indefiniteness of the gravitational action", Nucl. Phys. B 138, 141 (1978).

[14] K. Schleich, "Conformal rotation in perturbative gravity", Phys. Rev. D 36, 2342 (1987).

[15] S. W. Hawking, "The path-integral approach to quantum gravity", in: S. W. Hawking and W. Israel (eds.), General relativity: An Einstein Centenary Survey, Cambridge University Press (Cambridge, 1979).

[16] A. L. Besse, Einstein Manifolds, Springer (Berlin, Heidelberg, 1973).

[17] E. Baum, "Zero cosmological constant from minimum action", Phys. Lett. B 133, 185 (1983).

[18] S. W. Hawking, "The cosmological constant is probably zero", Phys. Lett. B 134, 403 (1984).

[19] A. Vilenkin, "Quantum cosmology and the initial state of the universe", Phys. Rev. D 37, 888 (1988).

[20] A. Lukas, "The No-Boundary Wave Function and the Duration of the Inflationary Period", preprint gr-qc/9409012. 
[21] S. W. Hawking, "Spacetime foam", Nucl. Phys. B 144, 349 (1978).

[22] M. Gasperini and G. Ummarino, "Adiabatic perturbations and minimal size of the de Sitter vacuum", Phys. Lett. B 266, 275 (1991).

[23] S. A. Hayward, "Signature change in general relativity", Class. Quantum Grav. 9, 1851 (1992).

[24] S. A. Hayward, "On cosmological isotropy, quantum cosmology and the Weyl curvature hypothesis", Class. Quantum Grav. 10, L7 (1993).

[25] M. Kossowski and M. Kriele, "Smooth and discontinuous signature type change in general relativity", Class. Quantum Grav. 10, 2363 (1993).

[26] M. Kossowski and M. Kriele, "Signature type change and absolute time in general relativity", Class. Quantum Grav. 10, 1157 (1993).

[27] M. Kossowski and M. Kriele, "Transverse, type changing, pseudo riemannian metrics and the extendability of geodesics", Proc. R. Soc. Lond. A 444, 297 (1994).

[28] M. Kossowski and M. Kriele, "The Einstein equation for signature type changing spacetimes", Proc. R. Soc. Lond. A 446, 115 (1994).

[29] M. Kriele and J. Martin, "Black holes, cosmological singularities and change of signature", preprint $\mathrm{gr-qc/9411063.}$

[30] F. Embacher, "Actions for signature change", University Vienna preprint UWThPh-1995-1, also preprint Er-qc/9501004, to appear in Phys. Rev. D (1995).

[31] A. Vilenkin, "Creation of universes from nothing", Phys. Lett. B 117, 25 (1982).

[32] I. Klebanov, L. Susskind and T. Banks, "Wormholes and the cosmological constant", Nucl. Phys. B 317, 665 (1989).

[33] G. W. Gibbons and M. J. Perry, "Quantizing gravitational instantons", Nucl. Phys. B 146, 90 (1978).

[34] A. Vilenkin, "Quantum creation of universes", Phys. Rev. D 30, 509 (1984). 
[35] A. D. Linde, "Quantum creation of inflationary universe", Nuovo Cimento 39, 401 (1984).

[36] A. Vilenkin, "Boundary conditions in quantum cosmology", Phys. Rev. D 33, 3560 (1986).

[37] P. G. O. Freund and M. A. Rubin, "Dynamics of dimensional reduction", Phys. Lett. 97 B, 233 (1980).

[38] D. Bailin and A. Love, "Kaluza-Klein theories", Rep. Prog. Phys. 50, 1087 (1987).

[39] M. J. Duff, "Kaluza-Klein theory in perspective", Talk delivered at the "Oskar Klein Centenary Nobel Symposium", Stockholm, September 1994, preprint hepth/9410046.

[40] J. Preskill, "Wormholes in spacetime and the constants of nature", Nucl. Phys. B 323, 141 (1989).

[41] S. W. Hawking, "Wormholes and Non-simply Connected Manifolds", in: S. Coleman et al (eds.), Quantum cosmology and baby universes, World Scientific (Singapore, 1991).

[42] M. Kriele, private communication.

[43] R. C. Myers, "Higher-derivative gravity, surface terms, and string theory", Phys. Rev. D 36, 392 (1987).

[44] M. S. Madsen and J. D. Barrow, "De Sitter ground states and boundary terms in generalised gravity", Nucl. Phys. B 323, 242 (1989). 